\title{
The Value of Implementing Multidisciplinary Perinatal Care Conference in the Private Practice Setting
}

\author{
Robert C. Johnston, MD ${ }^{1}$ Sina Haeri, MD, MHSA ${ }^{2,3}$ Richard T. Hale, PhD ${ }^{2,3}$ William Lindsley, RDMS 2,3 \\ Annette McCormick, MD ${ }^{3}$ Eric Su, MD ${ }^{3}$
}

${ }^{1}$ University of California Irvine Medical Center, Orange, California

${ }^{2}$ Austin Maternal-Fetal Medicine, Austin, Texas

${ }^{3}$ St. David's Women's Center of Texas, Austin, Texas

Address for correspondence Sina Haeri, MD, MHSA, Austin MaternalFetal Medicine, 12200 Renfert Way, G-3, Austin, TX 78758

(e-mail: sinahaeri@gmail.com).

Am J Perinatol Rep 2017;7:e201-e204.

\begin{abstract}
Objective The objective of this study was to estimate the impact of multidisciplinary (Multi-D) perinatal care conference (PCC) implementation in the private practice setting.

Methods After the initial 12-month period following implementation of the monthly PCC by private maternal-fetal medicine and neonatology practitioners, conference attendees were asked to completed a modified version of the Attitudes Toward Health Care Teams Scale, involving 19 questions assessing their attitudes and opinions toward Multi-D team care on a five-point Likert's scale.

Results Of the 51 average attendees to the PCC, 82.3\% completed the survey. A majority of respondents agreed that Multi-D team care resulted in improved care for

Keywords

- multidisciplinary

- perinatal

- care conference

- high risk

- fetal patients and family, was not overly complex to coordinate, and resulted in significant job satisfaction and improved medical knowledge.

Conclusion Multi-D care is an effective approach to the complicated needs of maternal-fetal medicine patients which may lead to improved patient and family outcomes, high provider satisfaction, and can easily be implemented and utilized within a private practice or community hospital setting.
\end{abstract}

A significant number of pregnancies every year are considered high risk. These include, but are not limited to complications such as hypertension, diabetes, multifetal gestation, advanced maternal age and congenital fetal anomalies. ${ }^{1,2}$ For instance, 6 to $8 \%$ of pregnant women in the United States have high blood pressure, and between 2 and $10 \%$ suffer from gestational diabetes. Approximately 1 in every 880 U.S. births in 2014 was a triplet or higher order gestation. ${ }^{3}$ As more couples choose to delay childbearing, the number of women aged 35 to 39 and 40 to 44 years has steadily increased across the United States since 1980, and now account for 11.0 and 2.3 per 1,000 births, respectively. ${ }^{4}$ Finally, approximately 1 in every 33

received

May 13, 2017

accepted after revision

September 26, 2017
DOI https://doi.org/

10.1055/s-0037-1608640. ISSN 2157-6998. infants in the United States is born with a birth defect, and account for $20 \%$ of all infant deaths. ${ }^{5}$

Maternal-fetal medicine (MFM) specialists often encounter these complex patients and are tasked with coordinating their multidisciplinary (Multi-D) prenatal, intrapartum, and neonatal care. This includes preconception care for women with medical or genetic risk factors or prior adverse pregnancy outcomes, referral to specialists for various obstetric, fetal, medical or surgical complications, intrapartum consultations resulting from complications of labor or delivery, and referral to appropriate consultants for ongoing management of complex issues in the postpartum state. ${ }^{6}$ However, caring for the
Copyright $\odot 2017$ by Thieme Medical Publishers, Inc., 333 Seventh Avenue, New York, NY 10001, USA. Tel: +1(212) 584-4662.
License terms

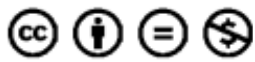


unborn fetus with anomalies is often even more challenging. Numerous management questions, from ethical to surgical to logistical often plague not only the treatment team but also the parents whose lives will be forever changed by their interactions with the team. ${ }^{7}$ A Multi-D approach involving MFM, neonatology, various pediatric surgical subspecialists, cardiology, anesthesiology, palliative care, social work, case management, and various institutional support staff members is often assembled to care for complex cases. Multi-D team care is now routinely employed across the United States for certain birth defects, such as cleft lip with or without cleft palate. ${ }^{8}$ Although there is literature regarding the effectiveness of Multi-D team approaches regarding outcomes, little is known about the attitudes and opinions of health care providers toward the Multi-D care they provide. The purpose of this study was to assess the attitudes and opinions of health care providers routinely involved in a Multi-D team care model within a private practice setting at a high-volume community hospital.

\section{Materials and Methods}

In 2014, a monthly Multi-D perinatal care conference (PCC) was established at St. David's Women's Center of Texas (large community hospital in Austin, TX) by the private MFM and neonatology practitioners. The conference structure consists of (1) presentation of new fetal anomaly cases by MFM providers from Austin MFM (first 20 minutes), (2) presentation of complicated neonatal cases and follow-up of previously presented fetal cases by neonatology (second 20 minutes), and (3) review of impending high-risk maternal cases requiring Multi-D care by other specialties including anesthesiology, cardiology, and social services (last 20 minutes). The invitations to attend were provided to medical staff within the St. David's Healthcare system, as well as pediatric subspecialists within the immediate market. Invitation to view the conference via the web was provided to referring practices and facilities beyond the immediate Austin area.

To assess the effectiveness of the PCC, a survey, based on a revised version of the Attitudes Toward Health Care Teams Scale, was created by adding two additional statements: "The PCC is improving my medical knowledge" and "The PCC is achieving the goal of creating a more complete approach to managing the care of our patients." ${ }^{\prime 9}$ The Attitudes Toward Health Care Teams Scale was created in an effort to measure aspects of interprofessional collaboration, which is "a process that aims to provide effective health services through teamwork among professionals from different backgrounds." ${ }^{9}$ The instrument contains a total of 19 questions, 18 of which are statements, participants are asked to respond with their opinion based on a five-point Likert's scale. ${ }^{10}$ The Likert's scale was created using 0 to represent "strongly disagree," 1 representing "disagree," 2 representing "neutral," 3 representing "agree," and 4 representing "strongly agree." The survey was administered via a third-party service, SoGo Survey (www. sogosurvey.com, Herndon, VA). Participants who attend our institution's monthly PCC were invited to complete the survey via e-mail and had the option of completing the survey anonymously. ${ }^{10,11}$ The invitation was sent by our health system's continuing medical education department to the invitee mail list outlined earlier. Due the anonymity of the assessment tool, we were not aware of the details surrounding the respondents' attendance record. Results from the responses were exported to an Excel document and reported as descriptive data. The study was approved by our Institutional Review Board.

\section{Results}

The monthly PCC averaged 51 participants per meeting from inception in 2014 through July 2015 when the survey assessment was sent to participants. Of the invitees, 42 $(82.3 \%)$ responded to the survey. The participants included 11 physicians (26\%), 15 nurses (35.7\%), 14 sonographers (33.3\%), and 2 individuals self-reported as other in terms of job title (4.8\%). In terms of age of respondents, $14.3 \%$ were between 21 and 30 years, 38.1\% were between 30 and 40 years, and $47.6 \%$ were 40 years or older. To avoid skewing the perception of the participants, the organizing MFM and neonatology providers were excluded from the survey as they actively participated in the organization and presentation of the conference on a regular basis.

A majority (64.3\%) of respondents answered that they strongly agree with the statement "Patients/clients receiving interprofessional care are more likely than others to be treated as whole persons." No respondents disagreed with that statement. When considering statements regarding the time and resources required to plan interprofessional care team processes, respondents' answers were more diverse, with $21.4 \%$ strongly disagreeing with the statement, "Developing an interprofessional patient/client care plan is excessively time consuming," $47.6 \%$ disagreeing with the statement, $7.1 \%$ agreeing with the statement, and another $7.1 \%$ strongly agreeing with the statement. However, most respondents (78.6\%) either disagreed or strongly disagreed that "In most instances, the time required for interprofessional consultations could be better spent in other ways." Respondents also reported significant job satisfaction in interprofessional approaches, as $88.1 \%$ of respondents either agreed or strongly agreed that "Working in an interprofessional environment keeps most health professionals enthusiastic and interested in their jobs."

Respondents tended to agree that efficiency of care was improved with interprofessional approaches-92.2\% of respondents either agreed or strongly agreed that "the interprofessional approach makes the delivery of care more efficient." In addition, all respondents either agreed or strongly agreed that interprofessional care helps decrease medical errors, with $66.7 \%$ of respondents strongly agreeing and $33.3 \%$ of respondents agreeing with the statement, "Developing a patient/client care plan with other team members avoids errors in delivering care." Respondents also largely felt that interprofessional care was not overly complex in nature, with $90.2 \%$ of respondents either disagreeing or strongly disagreeing with the statement, "Working in an interprofessional manner unnecessarily complicates things most of the time."

When considering the impact of interprofessional care plans on the emotional and financial needs of patients, $52.4 \%$ 
of respondents agreed and 33.3\% of respondents strongly agreed that "Health professionals working as teams are more responsive than others to the emotional and financial needs of patients/clients." Likewise, respondents largely agreed that interprofessional care plans transcend patient care to also address the needs of family members and caregivers$50.0 \%$ of respondents agreed and $38.1 \%$ of respondents strongly agreed with the statement, "The interprofessional approach permits health professionals to meet the needs of family caregivers as well as patients."

Multi-D care also enhanced respondents' understanding of other health care professionals' roles within the care team. Thirty-one percent of respondents agreed and $64.3 \%$ of respondents strongly agreed with the statement, "Having to report observations to a team helps team members better understand the work of other health professionals." When considering the transition from hospital care to home following discharge, $31.0 \%$ of respondents agreed with and $50.0 \%$ of respondents strongly agreed that, "Hospital patients who receive interprofessional team care are better prepared for discharge than other patients."

Respondents had similar views with regard to communication within interprofessional care teams, with $31.0 \%$ of respondents agreeing and $64.3 \%$ of respondents strongly agreeing that "Team meetings foster communication among team members from different professions or disciplines." The last two questions were added to the instrument to assess respondents' opinions related to the PCC specifically. The majority of respondents believed that the conference achieved its primary goal, as evidenced by $88.0 \%$ of respondents either agreeing or strongly agreeing with the statement, "The PCC is achieving the goal of creating a more complete approach to managing the care of our patients." Finally, respondent answers suggested that the vast majority consider interprofessional team approaches to be associated with aggregate increases in medical knowledge, with $33.3 \%$ of respondents agreeing and $64.3 \%$ of respondents strongly agreeing with the statement, "The PCC is improving my medical knowledge."

\section{Discussion}

Our survey of providers who regularly attend our monthly PCCs revealed many positive attitudes and opinions toward interprofessional care of the maternal-fetal dyad. Providers' responses suggested that the time spent involved in interdisciplinary team care improved their job satisfaction as well as their medical knowledge, and resulted (in their opinion) in meaningful outcomes with fewer medical errors and more complete care of the patient. In addition, providers responded that interprofessional care approaches transcended basic medical care by also addressing the social, financial, and emotional needs not only of their patients but of their families and caretakers as well.

Improvements in technology, genetic screening, and access to medical care has facilitated improved diagnosis and-in some cases-treatment of fetal anomalies, resulting in increasingly complex care of the fetal patient. Initially adopted from the concept of tumor board for cancer care, the perinatal conference, sometimes referred to as "fetal board," is the Multi-D approach to the abnormal fetal patient or complex mother. ${ }^{12,13}$ Studies have demonstrated several improved outcomes as a result of a Multi-D approach to complex maternal-fetal care, including changes in diagnosis, antenatal and postnatal management, timing of delivery, improvement in emotional, social and financial family well-being, and implementation of protocols that improve long-term outcomes. ${ }^{12-17}$

In 2011, the American College of Obstetricians and Gynecologists recommended that the organization and governance of centers involved in fetal intervention should involve a Multi-D team of health care providers. ${ }^{18}$ For several reasons, MFM specialists are well suited to coordinate MultiD care of the complex maternal-fetal dyad: they are often the first member of the team to interact with the patient and therefore possess a unique opportunity to establish rapport and trust with the family, ${ }^{8}$ and, by nature of their subspecialty training and knowledge of abnormal maternal and neonatal physiology may refine the fetal diagnosis, alter the antepartum management or guide the intrapartum process with the help of additional subspecialty providers.

There are little data regarding the prevalence of formalized Multi-D team care approaches across the United States. A recent survey of 29 U.S. fetal care centers demonstrated that the majority are administered by MFM specialists. ${ }^{19}$ Although there are undoubtedly more medical centers across the country that likely employ regular Multi-D team care processes, the current lack of a standardized assessment tool makes ascertainment of these processes difficult. With this knowledge gap in mind, we have shown that health care providers involved in a monthly PCC believe Multi-D care approaches to be effective, pragmatic, and may result in improved patient, family, and provider satisfaction. Although compelling for the implementation of a Multi-D care approach to complex maternal-fetal patients, our results should be taken in the context of our single-site institution, relatively small sample size, and lack of preimplementation assessment. Future research should include an assessment of the effectiveness of a Multi-D team approach from a patient and patient-family perspective. The creation of a standardized tool for assessing the effectiveness of a Multi-D team approach will hopefully assess the true goal of optimal and complete patient care.

\section{Conflict of Interest}

The authors report no conflicts of interest.

Financial Support

There was no financial support for this study.

\section{References}

1 What are the factors that put a pregnancy at risk? Eunice Kennedy Shriver National Institute of Child Health and Human Development. Updated June 17, 2013. Available at: https://www.nichd.nih.gov/ health/topics/high-risk/conditioninfo/pages/factors.aspx. Accessed August 24, 2016 
2 Reproductive Health. Centers for Disease Control and Prevention. June 17, 2016. Available at: http://www.cdc.gov/reproductivehealth/maternalinfanthealth//PregComplications.htm. Accessed August 24, 2016

3 Martin JA, Osterman MJ, Thoma ME. Declines in Triplet and Higher-order Multilpe Births in the United States, 1998-2014. NCHS Data Brief No. 243. Centers for Disease Control and Prevention. April, 2016. Available at: http://www.cdc.gov/nchs/products/databriefs/db243.htm. Accessed August 24, 2016

4 Mathews TJ, Hamilton BE. First Births to Older Women Continue to Rise. NCHS Data Brief No. 152. Centers for Disease Control and Prevention. May, 2014. Available at: http://www.cdc.gov/nchs/ data/databriefs/db152.htm. Accessed August 24, 2016

5 Data \& Statistics. Centers for Disease Control and Prevention. Updated February 29, 2016. Available at: http://www.cdc.gov/ ncbddd/birthdefects/data.html. Accessed August 24, 2016

6 Sciscione A, Berghella V, Blackwell S, et al; Society for MaternalFetal Medicine (SMFM). Society for maternal-fetal medicine (SMFM) special report: the maternal-fetal medicine subspecialists' role within a health care system. Am J Obstet Gynecol 2014; 211(06):607-616

7 Bijma HH, Van der Heide A, Wildschut HI, Van der Maas PJ, Wladimiroff JW. Impact of decision-making in a multidisciplinary perinatal team. Prenat Diagn 2007;27(02):97-103

8 James JN, Schlieder DW. Prenatal counseling, ultrasound diagnosis, and the role of maternal-fetal medicine of the cleft lip and palate patient. Oral Maxillofac Surg Clin North Am 2016;28(02):145-151

9 Heinemann GD, Schmitt MH, Farrell MP, Brallier SA. Development of an Attitudes Toward Health Care Teams Scale. Eval Health Prof 1999;22(01):123-142
10 Kaplan R, Saccuzzo D. Psychological Testing: Principles, Applications, and Issues, 7th ed. Belmont, CA: Wadsworth, Cengage Learning; 2009

11 Landy FJ, Conte JM. Work in the 21st Century. 3rd ed. Hoboken, NJ: John Wiley \& Sons, Inc.; 2010

12 Porter KB, Wagner PC, Cabaniss ML. Fetal Board: a multidisciplinary approach to management of the abnormal fetus. Obstet Gynecol 1988;72(02):275-278

13 Luks FI, Carr SR, Feit LR, Rubin LP. Experience with a multidisciplinary antenatal diagnosis and management model in fetal medicine. J Matern Fetal Neonatal Med 2003;14(05):333-337

14 Raboei EH. The role of the pediatric surgeon in the perinatal multidisciplinary team. Eur J Pediatr Surg 2008;18(05):313-317

15 Han HH, Choi EJ, Kim JM, Shin JC, Rhie JW. The importance of multidisciplinary management during prenatal care for cleft lip and palate. Arch Plast Surg 2016;43(02):153-159

16 Lim AS, Stewart K, Abramson MJ, Walker SP, Smith CL, George J. Multidisciplinary Approach to Management of Maternal Asthma (MAMMA): a randomized controlled trial. Chest 2014;145(05): 1046-1054

17 Aite L, Trucchi A, Nahom A, Spina V, Bilancioni E, Bagolan P. Multidisciplinary management of fetal surgical anomalies: the impact on maternal anxiety. Eur J Pediatr Surg 2002;12(02):90-94

18 American College of Obstetricians and Gynecologists Committee on Ethics, American Academy of Pediatrics Committee on Bioethics. Committee Opinion Number 501: Maternal-Fetal Intervention and Fetal Care Centers. The American College of Obstetricians and Gynecologists; 2011

19 Kett JC, Woodrum DE, Diekema DS. A survey of fetal care centers in the United States. J Neonatal Perinatal Med 2014;7(02):131-135 